\title{
Corrigenda
}

\section{Localized Bicaudal-C RNA encodes a protein containing a KH domain, the RNA binding motif of FMR1}

Michèle Mahone, Emma E.Saffman and
Paul F.Lasko

The EMBO Journal, 14, 2043-2055, 1995
In the above paper, the nucleotide and predicted amino acid sequence of the Bic-C gene was published as Figure 5. Subsequently, sequencing of additional mutant Bic-C alleles in the authors' laboratory and the completion of the genomic sequence surrounding $B i c-C$ by the Berkeley Drosophila Genome Project (BDGP) have indicated a number of errors in the sequence. The revised sequence, which, with the exception of one nucleotide in the $5^{\prime}$ UTR, agrees entirely with that reported by the BDGP, is published below as a revised Figure 5.

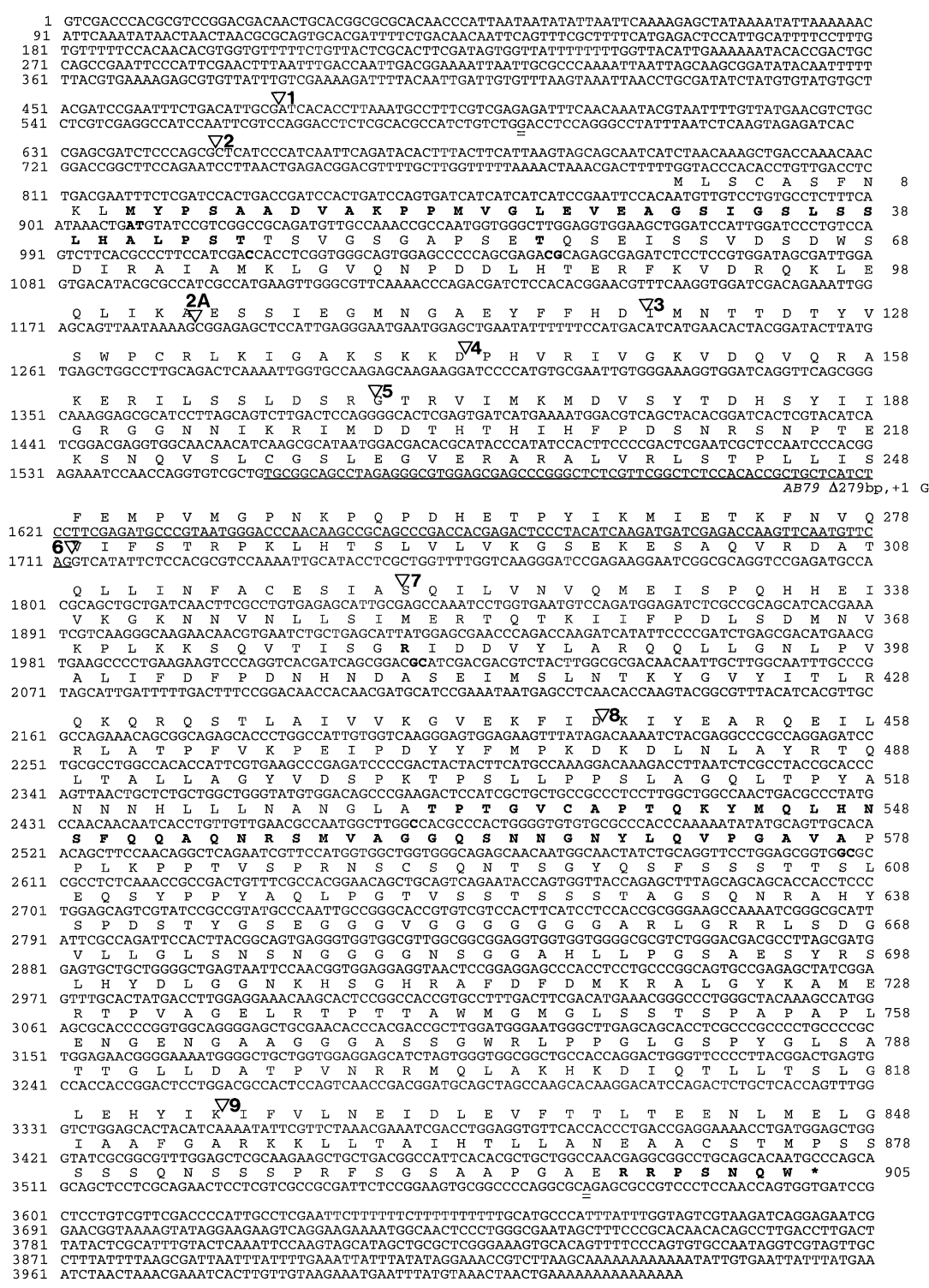

Fig. 5. Corrected nucleotide and predicted amino acid sequence of the Bic-C gene. Changed amino acids and nucleotides are in bold type, and two deletions of two nucleotides each are adjacent to residues which are double underlined. These changes do not affect the nature of the five KH domains nor the region of similarity with Eph-family tyrosine kinases, but note that the protein no longer contains a predicted transmembrane domain at the carboxyl terminus. Sequencing of the corresponding genomic DNA showed that the gene is interrupted by 10 introns, whose positions are marked by arrows. The lengths of the introns are as follows: intron 1, 548 nucleotides (nt); intron 2, $574 \mathrm{nt}$; intron $2 \mathrm{~A}, 61 \mathrm{nt}$; intron 3, $316 \mathrm{nt}$; intron 4, $62 \mathrm{nt}$; intron 5, $65 \mathrm{nt}$; intron 6, $444 \mathrm{nt}$; intron 7, $61 \mathrm{nt}$; intron 8, $193 \mathrm{nt}$; intron 9, $228 \mathrm{nt}$. The Bic-C $C^{A B 79}$ deletion removes the underlined nucleotides and the first 120 nucleotides of intron 6. In Bic- $C^{R U 35}$ the $\mathrm{G}$ at position 1761 is replaced by an A. In addition, sequence of the P1 clone DS00913 performed by the Berkeley Drosophila Genome Project (DDBJ/EMBL/GenBank accession numbers L43436-L43440) suggests that the poly(A) stretch at the end of our cDNA represents a site of internal priming, as a consensus polyadenylation signal is present 67 nucleotides downstream. These corrections to the genomic and cDNA sequences have been submitted to DDBJ/EMBL/GenBank databases, accession number U15928. 


\section{A purified mariner transposase is sufficient to mediate transposition in vitro}

\section{David J.Lampe, Mair E.A.Churchill and Hugh M.Robertson}

The EMBO Journal, 15, 5470-5479, 1996

In Figure 5B the authors erroneously represented the $5^{\prime}$ DNA cleavage pattern produced by Himarl transposase as occurring from two nucleotides within the element outward. The correct cleavage pattern is shown below in a corrected Figure 5B and occurs from three nucleotides within the element outward as is clear from Figure 5A. This mistake was carried over into the Discussion (pages 5475-5476) and led to the erroneous conclusion that the termini of Himarl are cleaved in a manner more similar to that of the $T c 1$ transposable element rather than that of another mariner-family element, Mos 1 . From these data the authors would now predict that all mariner-family elements share the property of producing predominantly three nucleotide $3^{\prime}$ overhangs upon excision.

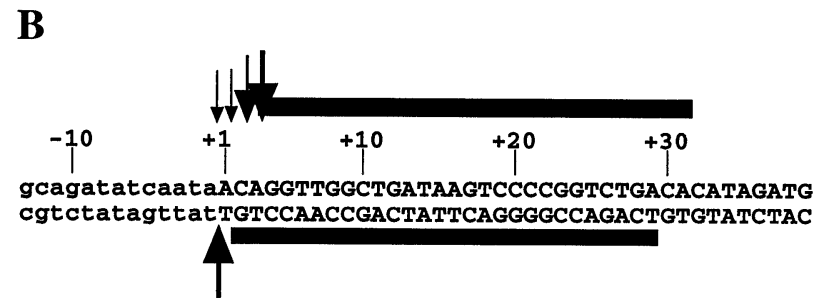

\section{Erratum}

Receptor co-operation in retrovirus entry: recruitment of an auxiliary entry mechanism after retargeted binding

Sandrine Valsesia-Wittmann, Frances J.Morling, Theodora Hatziioannou, Stephen J.Russell and François-Loïc Cosset

The EMBO Journal, 16, 1214-1223, 1997

In the above paper, due to a publisher's error, the affiliation for François-Loïc Cosset was incorrect. The correct affiliation is:

Centre de Génétique Moléculaire et Cellulaire, CNRS UMR5534, UCB Lyon-I, 43 Boulevard du 11 Novembre 1918, 69622 Villeurbanne Cedex, France

and not Cambridge Centre for Protein Engineering, UK.

\section{Retraction}

Ribozyme-mediated cleavage of the MDR-1 transcript restores chemosensitivity in previously resistant cancer cells

Michael Kiehntopf, Marion A.Brach, Thomas Licht, Simone Petschauer, Leonid Karawajew, Carsten Kirschning and Friedhelm Herrmann

The EMBO Journal, 13, 4645-4652, 1994

Following the admission of systematic fabrication of results by co-authors Marion Brach and Michael Kiehntopf, and at the request of the Dean of the Medical Faculty of the University of Ulm, Germany, the editorial board of The EMBO Journal wish to retract this paper. 